Surveys in Differential Geometry XV

\title{
Subgroups of Depth Three
}

\author{
Sebastian Burciu and Lars Kadison
}

\begin{abstract}
A subalgebra pair of semisimple complex algebras $B \subseteq$ $A$ with inclusion matrix $M$ is depth two iff $M M^{t} M \leq n M$ for some positive integer $n$ and all corresponding entries. If $A$ and $B$ are the group algebras of finite group-subgroup pair $H<G$, the inductionrestriction table equals $M$ and $S=M M^{t}$ satisfies $S^{2} \leq n S$ iff the subgroup $H$ is depth three in $G$; similarly depth $n>3$ by successive right multiplications of this inequality with alternately $M$ and $M^{t}$. We show that a Frobenius complement in a Frobenius group is a nontrivial class of examples of depth three subgroups. A tower of Hopf algebras $A \supseteq B \supseteq C$ is shown to be depth-3 if $C \subseteq \operatorname{core}(B)$; and this is also a necessary condition if $A, B$ and $C$ are group algebras.
\end{abstract}

\section{Introduction}

Induction of characters from a subgroup to a group is a useful technique for completing character tables $[\mathbf{8}]$ found by nineteenth century algebraists. At about the same time, Frobenius discovered reciprocity, which in modern terms states that induction is naturally isomorphic to coinduction of $G$-modules, either forming an adjoint pair with the restriction functor, and applies to any Frobenius extension of algebras.

Finite index subfactors are a certain type of Frobenius extension, where an analytic notion of finite depth was discovered in connection with classification, with depth two being part of a remarkable type of Galois theory of paragroups. The notion of finite depth was eventually made algebraic and applied to Frobenius extensions; later, depth two and its Galois theory of quantum groupoids and Hopf algebroids were exposed in simplest terms for ring extensions (see [18] for an application to J. Roberts field algebra construction $[\mathbf{1 6}])$.

It was noted in $[\mathbf{1 0}]$ that the notion of depth two applies to characters of a finite group and subgroup pair via complex group algebras: a subgroup 
is depth two if no new constituents arise when inducing-restricting-inducing a character as compared with inducing just one time. By means of general theory in one direction and Mackey theory in the other, depth two subgroup is shown to be precisely a normal subgroup [10]. (A similar statement is true for semisimple Hopf $\mathbb{C}$-subalgebras [4].) In this paper we generalize this approach to depth two subgroup to a semisimple subalgebra pair, giving a condition in terms of inclusion matrix [7], which is the same as a inductionrestriction table [1] up to a permutation change of basis. The depth two condition is essentially that the cube of the inclusion matrix is less entrywise than a multiple of the inclusion matrix, noted more precisely in the abstract and Proposition 1.2 below.

In [11] it was shown that finite depth Frobenius extension has a simplified definition in terms of a generalization of depth two to a tower of three algebras in the Jones tower. In this paper we extend a particular case of an embedding theorem in $[\mathbf{1 1}]$ to characterization of certain finite depth separable Frobenius extension in terms of depth two extension in Jones tower (see Theorems 2.1 and 2.5 below). Then one may check that a subgroup is depth three or more by comparing cube of symmetric matrix $\mathcal{S}$ of inner products of induced irreducible characters with multiples of $\mathcal{S}$ (see Prop. 2.2). In somewhat the same spirit, Corollary 2.9 below implies that a subgroup is depth three if no new constituents arise from applying restriction-induction one extra time to a character.

Although amusing to test for depth three property from character tables of groups and non-normal subgroup, it is not clear from this definition what precisely a depth three subgroup is. A number of proposals to remedy this are given below: depth three quasi-bases are given in Theorem 2.10, a characterization of certain depth three Frobenius extension in terms of similar bimodules, tensor-square and overalgebra in Theorem 2.7, and a class of examples in Section 3, a Frobenius group and its Frobenius complement. Even the notion of depth-3 tower of algebras may be viewed as an alternative to defining finite depth in terms of iterated endomorphism algebra extensions (perhaps applied instead to an iteration of another useful construction). Depth-3 towers of finite group algebras are completely classified in Theorem 1.1 following the spirit of [11]. Depth-3 towers of Hopf algebras are also considered at the end of the second section. A tower of Hopf algebras $A \supseteq B \supseteq C$ is depth-3 if $C \subseteq \operatorname{core}(B)$ (see subsection 1.6 for the definition of the core of a Hopf subalgebra). Using then notion of kernel of a module introduced in [3] we formulate a conjecture on the core of a Hopf subalgebra. This conjecture would imply that the condition $C \subseteq \operatorname{core}(B)$ is also a necessary condition for the Hopf algebra tower $A \supseteq B \supseteq C$ to be depth-3 (which is true for group algebras by the Theorem 1.1 below).

Although our algebras are often over the complex numbers, we have tried to write this paper in a change-of-characteristic-friendly way. 


\section{Preliminaries on depth two extensions}

All algebras in this paper are associative algebras (not necessarily commutative) over a field $k$. Given an $(A, A)$-bimodule $M$, we let $M^{A}$ denote the $A$-central elements $\{m \in M \mid \forall a \in A, a m=m a\}$.

Two $r \times s$ integer matrices $M$ and $N$ satisfy $M \leq N$ if each of the coefficients $m_{i j} \leq n_{i j}$ : this property is independent of permutation of bases. Note that if $X$ is a third $q \times r$ matrix of non-negative integers, then $X M \leq$ $X N$; if $\mathrm{X}$ is $s \times q$, then $M X \leq N X$. We say $M$ is strictly positive if all entries $m_{i j}>0$.

1.1. Frobenius extensions. A Frobenius extension $A \mid B$ is an extension of associative algebras where the natural bimodule ${ }_{B} A_{A}$ is isomorphic to the $(B, A)$-bimodule $\operatorname{Hom}\left(A_{B}, B_{B}\right)$ (of right $B$-module homomorphisms) given by $(b \cdot f \cdot a)(x)=b f(a x)$ for $a, x \in A, b \in B, f \in \operatorname{Hom}\left(A_{B}, B_{B}\right)$. This is equivalent to the existence of a mapping $F \in \operatorname{Hom}\left({ }_{B} A_{B},{ }_{B} B_{B}\right)$ with dual bases $\left\{x_{i}\right\}_{i=1}^{n}$ and $\left\{y_{i}\right\}_{i=1}^{n}$ such that $\sum_{i=1}^{n} F\left(a x_{i}\right) y_{i}=a$ and $\sum_{i=1}^{n} x_{i} F\left(y_{i} a\right)=$ $a$ for all $a \in A$ : we call the data system $F$ a Frobenius homomorphism with dual bases $\left\{x_{i}\right\},\left\{y_{i}\right\}$.

For example, a group algebra $A=k[G]$ is a Frobenius extension of any subgroup algebra $B=k[H]$, where $H \leq G$ is a subgroup of finite index $[G$ : $H]=n$. For if $\left\{g_{i}\right\}_{i=1}^{n}$ denotes left coset representatives of $H$ in $G$, where $g_{1}=1_{G}$, a Frobenius system is given by $x_{i}=g_{i}^{-1}, y_{i}=g_{i}$ with bimodule projection given by $\left(n_{h g_{i}} \in k\right)$

$$
F\left(\sum_{i=1}^{n} \sum_{h \in H} n_{h g_{i}} h g_{i}\right)=\sum_{h \in H} n_{h} h
$$

a routine exercise.

A Frobenius extension $A \mid B$ enjoys isomorphic tensor-square and endomorphism ring as $(A, A)$-bimodules. We note that $A \otimes_{B} A \cong$ End $A_{B}$ via $x \otimes_{B} y \mapsto \lambda(x) \circ F \circ \lambda(y)$. Also $A \otimes_{B} A \cong$ End $_{B} A$ via $x \otimes y \mapsto \rho(y) \circ F \circ$ $\rho(x)[\mathbf{9}]$. Composing the two isomorphisms we obtain an anti-isomorphism End $A_{B} \rightarrow$ End $_{B} A$ given by $f \mapsto \sum_{i} F\left(-f\left(x_{i}\right)\right) y_{i}$, which restricts to an antiautomorphism on the subring $\operatorname{End}_{B} A_{B}$, and plays the role of antipode in case of depth two Frobenius extension defined below.

1.2. Separable extensions. If the characteristic of the ground field $k$ is coprime to $[G: H]=n$, then the extension of group algebras $A \mid B$ noted above is a separable extension: i.e. the multiplication map $\mu: A \otimes_{B} A \rightarrow$ $A$ is a split $(A, A)$-epimorphism. The image of $1_{A}$ under a section $A \rightarrow$ $A \otimes_{B} A$ is a separability element $e=\sum_{i=1}^{n} e_{i} \otimes_{B} f_{i}$ satisfying $a e=e a$ for all $a \in A$ and $\mu(e)=\sum_{i=1}^{n} e_{i} f_{i}=1_{A}$, which characterizes separable extension. 
Notice that

$$
\frac{1}{[G: H]} \sum_{i=1}^{n} g_{i}^{-1} \otimes_{B} g_{i}
$$

is a separability element for the group algebras $A$ over $B$.

In the situation that $C \supseteq A \supseteq B$ is a tower of algebras and $A \mid B$ is a separable extension, the canonical epi $C \otimes_{B} C \rightarrow C \otimes_{A} C$ given by $c_{1} \otimes_{B} c_{2} \mapsto$ $c_{1} \otimes_{A} c_{2}$ splits. A section for this mapping is of course given by $c_{1} \otimes_{A} c_{2} \mapsto$ $\sum_{i=1}^{n} c_{1} e_{i} \otimes_{B} f_{i} c_{2}$.

1.3. Depth-3 towers of algebras. A tower of three algebras $A \supseteq B \supseteq$ $C$, where $C$ is a unital subalgebra of $B$ which is in turn unital subalgebra of $A$, is said to be right depth-3, or right $\mathrm{d}-3$, if there is a complementary $(A, C)$-bimodule $P$ and $n \in \mathbb{N}$ such that

$$
A \otimes_{B} A \oplus P \cong A^{n}
$$

as natural $(A, C)$-bimodules. Equivalently, there is a split $(A, C)$-bimodule epimorphism from a finite direct sum of $A$ with itself to $A \otimes_{B} A$ ( $P$ is the kernel of such an epi).

Left d-3 towers are defined oppositely, so that $A \supseteq B \supseteq C$ is left d-3 iff the tower of opposite algebras $A^{\mathrm{op}} \supseteq B^{\mathrm{op}} \supseteq C^{\mathrm{op}}$ is right d-3. It has been noted in $[\mathbf{1 1}, \mathbf{5}]$ that if $A \mid B$ is a Frobenius, or quasi-Frobenius (QF, where isomorphisms above are replaced by similarity of bimodules) extension, then left $\mathrm{d}-3$ is equivalent with right $\mathrm{d}-3$ extension.

For example, a subnormal series of subgroups $G \triangleright N \triangleright H$ with corresponding group algebras $A \supseteq B \supseteq C$ (over any ground field) is a depth3 tower, since the normal closure $H^{G} \subseteq N^{G}=N$ and [11, Theorem 3.1] applies.

1.3.1. Depth-3 towers of semisimple algebras. Suppose a tower $A \supseteq B \supseteq$ $C$ of semisimple finite dimensional $k$-algebras is right $\mathrm{d}-3$. Tensoring 3 by $-\otimes_{C} M$ this implies that the following inequality:

$$
\left\langle M \uparrow_{C}^{A} \downarrow_{B}^{A} \uparrow_{B}^{A}, Q\right\rangle \leq n\left\langle M \uparrow_{C}^{A}, Q\right\rangle
$$

holds for any simple $C$-module $M$ and any simple $A$-module $Q$.

Using this relation a necessary and sufficient condition for a tower of groups to be depth-3 will be given in the next theorem. For $H$ a subgroup of $G$ let

$$
\operatorname{core}_{G}(H)=\cap_{g \in G}{ }^{g} H
$$

be the largest subgroup of $H$ which is normal in $G$. (Here ${ }^{g} H=g H g^{-1}$.)

Let $G \supseteq N \supseteq H$ be a tower of groups. Since $H^{G}$ is the subgroup of $G$ generated by the elements $g h g^{-1}$ with $g \in G$ and $h \in H$ note that $H \subseteq \operatorname{core}_{G}(N)$ if and only if $H^{G} \subseteq N$.

Theorem 1.1. A tower $G \supseteq N \supseteq H$ of groups is depth three if and only if $H \subset \operatorname{core}_{G}(N)$. 
Proof. If $H \subset \operatorname{core}_{G}(N)$ then $H^{G} \subseteq N$ and the proof of Theorem 3.1 from [11] applies.

Suppose now that the tower is depth-3. The above argument for the tower $k G \supseteq k N \supseteq k H$ of semisimple algebras implies that there is $n \in \mathbb{N}$ such that

$$
\left\langle\alpha \uparrow_{H}^{G} \downarrow_{N}^{G} \uparrow_{N}^{G}, \mu\right\rangle \leq n\left\langle\alpha \uparrow_{H}^{G}, \mu\right\rangle
$$

for any characters $\alpha \in \operatorname{Irr}(H)$ and $\mu \in \operatorname{Irr}(G)$.

Put $\mu=1_{G}$, the trivial character in the above inequality. Since $\left\langle\alpha \uparrow_{H}^{G}\right.$ , $\left.1_{G}\right\rangle=\left\langle\alpha, 1_{H}\right\rangle$ it follows that $\left\langle\alpha \uparrow_{H}^{G} \downarrow_{N}^{G} \uparrow_{N}^{G}, 1_{G}\right\rangle=0$ if $\alpha \neq 1_{H}$. By Frobenius reciprocity this implies that $\left\langle\alpha \uparrow_{H}^{G} \downarrow_{N}^{G}, 1_{N}\right\rangle=0$ if $\alpha \neq 1_{H}$.

On the other hand applying Mackey's theorem one has:

$$
\begin{aligned}
0=\left\langle\alpha \uparrow \uparrow_{H}^{G} \downarrow_{N}^{G}, 1_{N}\right\rangle & =\sum_{N g H \in N \backslash G / H}\left\langle{ }^{g} \alpha \downarrow_{\left.N \cap{ }_{H}{ }_{H} \uparrow_{N \cap{ }^{g} H}^{N}, 1_{N}\right\rangle}\right\rangle \sum_{N g H \in N \backslash G / H}\left\langle{ }^{g} \alpha \downarrow_{N \cap{ }^{g} H}^{g}, 1_{N \cap g_{H}}\right\rangle \\
& =\sum_{N g H \in N \backslash G / H}\left\langle\alpha \downarrow_{g^{-1} N \cap H}^{H}, 1_{g^{-1}{ }_{N \cap H}}\right\rangle \\
& =\sum_{N g H \in N \backslash G / H}\left\langle\alpha, 1_{g^{-1} N \cap H} \uparrow_{g^{-1} N \cap H}^{H}\right\rangle
\end{aligned}
$$

On the other hand using Frobenius reciprocity again one has

$$
\left\langle 1_{H}, 1_{g^{-1} N \cap H} \uparrow_{g^{-1} N \cap H}^{H}\right\rangle=\left\langle 1_{g^{-1} N \cap H}, 1_{g^{-1} N \cap H}\right\rangle=1
$$

Thus

$$
1_{g^{-1} N \cap H} \uparrow_{g^{-1}{ }_{N}^{H} \cap H}=1_{H}
$$

which implies that $H=g^{-1} N \cap H$ or $H \subset g^{-1} N=g^{-1} N g$. Thus $H \subset$ $\operatorname{core}_{G}(N)$.

1.4. Depth two algebra extensions. An algebra extension $A \supseteq B$ is defined to be right depth two (equivalently, subalgebra $B \subseteq A$ is $\mathrm{rD2}$ ) if the partially trivial tower $A \supseteq B \supseteq B$ is right d-3; similarly we define left D2 in terms of partially trivial left $\mathrm{d}-3$ tower.

It is obvious that a finite dimensional algebra $A$ is a depth two extension of its unit subalgebra $B=k 1_{A}$ : if $\operatorname{dim}_{k} A=n$, then of course ${ }_{A} A \otimes_{k} A \cong{ }_{A} A^{n}$. Similarly, we may show that if $C$ is a finite dimensional dimensional algebra, the tensor algebra $A=C \otimes B$ is a depth two extension of its subalgebra $B=1_{C} \otimes B$.

The main examples in the literature of depth two extension are HopfGalois extensions as well as its classical, weakened and pseudo- variants.

The defining Condition (3), with $B=C$, for right depth two extension is similar to the characterization of projective module as isomorphic to a direct summand of a free module. Like the derivation of projective bases for 
a projective module, we may derive from this condition right $\mathrm{D} 2$ quasi-bases for the right D2 extension $A \mid B$ as follows. For any ring extension, using the hom-tensor relation, note that $\operatorname{Hom}\left({ }_{A} A \otimes_{B} A_{B},{ }_{A} A_{B}\right) \cong \operatorname{End}_{B} A_{B}$. By evaluation at $1_{A}$ note that $\operatorname{Hom}\left({ }_{A} A_{B},{ }_{A} A \otimes_{B} A_{B}\right) \cong\left(A \otimes_{B} A\right)^{B}$.

Then the split epi from $\pi: A^{n} \rightarrow A \otimes_{B} A$ satisfies an equation $\pi \circ \sigma=$ $\operatorname{id}_{A \otimes_{B} A}$. We have $n$ standard split epis $A^{n} \rightarrow A$, which compose with $\pi$ and $\sigma$ to give the equation $\sum_{i=1}^{n} f_{i} \circ g_{i}=\mathrm{id}_{A \otimes_{B} A}$, where $f_{i} \in \operatorname{Hom}\left(A, A \otimes_{B} A\right)$ and $g_{i} \in \operatorname{Hom}\left(A \otimes_{B} A, A\right)$, to which we apply the simplifications noted above. Suppose $f_{i} \mapsto u_{i} \in\left(A \otimes_{B} A\right)^{B}$, while $g_{i} \mapsto \gamma_{i} \in \operatorname{End}_{B} A_{B}$ for each $i=1, \ldots, n$. As a consequence, we obtain for any $x, y \in A$ the identity

$$
x \otimes_{B} y=\sum_{i=1}^{n} x \gamma_{i}(y) u_{i}
$$

Note that an extension $A \mid B$ having elements $u_{i} \in\left(A \otimes_{B} A\right)^{B}$ and endomorphisms $\gamma_{i} \in$ End $_{B} A_{B}$ satisfying this identity, eq. (4), also implies that $A \mid B$ is right D2, since $A^{n} \rightarrow A \otimes_{B} A$ given by $\left(a_{1}, \ldots, a_{n}\right) \mapsto \sum_{i} a_{i} u_{i}$ is an $(A, B)$-epimorphism with section given by $x \otimes_{B} y \mapsto\left(x \gamma_{1}(y), \ldots, x \gamma_{n}(y)\right)$.

For example, a normal subgroup $N$ of index $n$ in any group $G$ (over any ground ring) is depth two with D2 quasi-bases given by $u_{i}=g_{i}^{-1} \otimes g_{i}$ and $\gamma_{i}(g)=F\left(g g_{i}^{-1}\right) g_{i}$ for coset representatives $\left\{g_{1}=e, g_{2}, \ldots, g_{n}\right\}$.

1.5. When inclusion matrix is depth two. Let the ground field $k=\mathbb{C}$ be the complex numbers when we consider semisimple algebras, which consequently become multi-matrix algebras (or split semisimple algebras). Suppose $B \subseteq A$ is a subalgebra pair of semisimple algebras. As one constructs an induction-restriction table for a subgroup $H$ in a finite group $G[\mathbf{1}, \mathrm{p}$. 166], we briefly review the procedure for generalizing to any pair of semisimple algebras (such as finite dimensional complex group algebras). Label the simples of $A$ by $V_{1}, \ldots, V_{s}$ and the simple modules of $B$ by $W_{1}, \ldots, W_{r}$. To obtain the $i$ 'th column restrict the $i$ 'th simple $A$-module $V_{i}$ to a $B$-module and express in terms of direct sum of simples

$$
V_{j} \downarrow_{B} \cong \oplus_{i=1}^{r} m_{i j} W_{i}
$$

We let $M$ be the $r \times s$-matrix, or table, with entries $m_{i j}: M=\left(m_{i j}\right)$. By a well-known generalization of Frobenius reciprocity, the rows give induction of the $B$-simples:

$$
W_{i} \uparrow^{A}=W_{i}^{A}=\oplus_{j=1}^{s} m_{i j} V_{j}
$$

since $W_{j}^{A}=W_{j} \otimes_{B} A$ and $V_{i} \downarrow_{B} \cong \operatorname{Hom}\left(A_{B}, V_{i}\right)$; i.e. if $\left[W_{j}^{A}, V_{i}\right]$ denotes the number of constituents in $W_{j}^{A}$ isomorphic to $V_{i}$, Frobenius reciprocity is given by

$$
\left[W_{i}^{A}, V_{j}\right]=m_{i j}=\left[W_{i}, V_{j} \downarrow_{B}\right]
$$

The matrix $M$ is also known as the inclusion matrix of $B$ in $A$ [7]. 
For example, the induction-restriction table (based on Frobenius reciprocity $\left.\left(\psi_{i}^{G}, \chi_{j}\right)_{G}=\left(\psi_{i}, \chi_{j} \downarrow_{H}\right)_{H}\right)$ for the standard embedding of permutation groups $S_{2} \leq S_{3}$ is given by

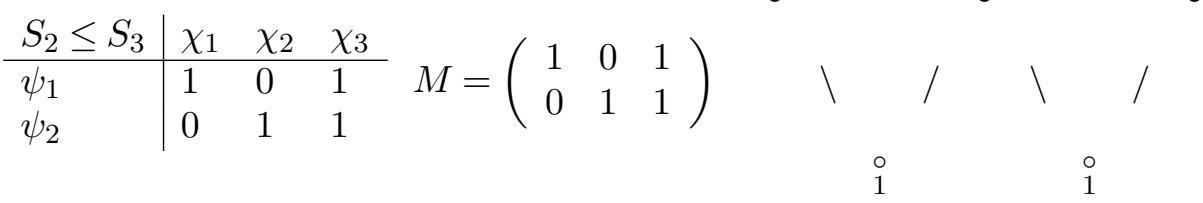

where $\psi_{1}=1_{H}, \chi_{1}=1_{G}$ denote the trivial characters, $\psi_{2}, \chi_{2}$ the sign characters, and $\chi_{3}$ the two-dimensional irreducible character of $S_{3}$. Note too the inclusion diagram or Bratteli diagram, a bicolored weighted multigraph [7].

For example, $1_{H}^{G}=\chi_{1}+\chi_{3}$ and $1_{H}^{G} \downarrow_{H}=2 \cdot 1_{H}+\psi_{2}$. Burciu [3] notes that a subgroup $H$ is normal in $G$ if and only if $1_{H}{ }^{G} \downarrow_{H}=[G: H] 1_{H}$. In [10] it is established that the notion of depth two subalgebra for subalgebra pair of complex group algebras is equivalent to the notion of normal subgroup.

Proposition 1.2. The inclusion matrix $M$ of a subalgebra pair of semisimple complex algebras $B \subseteq A$ satisfies

$$
M M^{t} M \leq n M
$$

for some positive integer $n$ if and only if $B$ is depth two subalgebra of $A$.

Proof. $(\Leftarrow)$ The depth two condition $A \otimes_{B} A \oplus P \cong A^{n}$ as natural $B$ - $A$-bimodules, becomes

$$
\left[W_{i}^{A} \downarrow_{B} \uparrow^{A}, V_{j}\right] \leq n\left[W_{i}^{A}, V_{j}\right]=n m_{i j}
$$

for all $i=1, \ldots, r$ and $j=1, \ldots, s$. But $W_{i}^{A}$ is given by row $i$ of $M$, or $e_{i} M$, where $e_{i}$ denotes row matrix with all zeroes except 1 in $i$ 'th column. Then $W_{i}^{A} \downarrow_{B}$ is given by $M\left(e_{i} M\right)^{t}=M M^{t} e_{i}^{t}$. Finally $W_{i}^{A} \downarrow_{B} \uparrow^{A}$ is given by $\left(M M^{t} e_{i}^{t}\right)^{t} M$, i.e. row $i$ of $M M^{t} M$.

$(\Rightarrow)$ If the inclusion matrix $M$ of semisimple subalgebra pair $B \subseteq A$ satisfies $M M^{t} M \leq n M$ for some $n \in \mathbb{Z}_{+}$, then $\left[\operatorname{Ind}_{B}^{A} \operatorname{Res}_{B}^{A} \operatorname{Ind}_{B}^{A} W_{i}, V_{j}\right] \leq$ $n\left[\operatorname{Ind}_{B}^{A} W_{i}, V_{j}\right]$ for all $B$-simples $W_{i}$ and $A$-simples $V_{j}$ (fix these orderings). Via unique module decomposition into simples, we find a monic natural transformation $\operatorname{Ind}_{B}^{A} \operatorname{Res}_{B}^{A} \operatorname{Ind}_{B}^{A} \hookrightarrow n \operatorname{Ind}_{B}^{A}$ from category $B$-Mod into $A$-Mod. Now $B, A$ and so $B^{o p} \otimes A$ are separable $\mathbb{C}$-algebras, so as in [[10], Theorem 2.1(6), pp. 3107-3108], we apply the natural monic to the right regular module $B_{B}$, apply the natural transformation property to all left multiplications $\lambda_{b}(b \in B)$, and note that $A \otimes_{B} A \hookrightarrow A^{n}$ splits by Maschke as $B$ - $A$-bimodule monic. Hence $A$ is depth two over its subalgebra $B$. 
1.6. Depth-3 tower of of Hopf algebras. For $B \subset A$ an extension of finite dimensional Hopf algebras, define $\operatorname{core}(B)$ to be the largest Hopf subalgebra of $B$ which is normal in $A$. It is easy to see that $\operatorname{core}(A)$ always exists (see also [3]). If $H \subset G$ is a group inclusion with $A=k G$ and $B=k H$ note that $\operatorname{core}(B)=k \operatorname{core}_{G}(H)$.

TheOREm 1.3. Suppose that $A \supseteq B \supseteq C$ is a tower of semisimple Hopf algebras. If $C \subset \operatorname{core}(B)$ then the tower is depth three.

Proof. Since $\operatorname{core}(B)$ is a normal Hopf subalgebra of $A$ it follows that the extension core $(B) \subset A$ is $D 2$ and therefore $A \otimes_{\operatorname{core}(B)} A$ is a direct summand of the bimodule ${ }_{A}\left(A^{n}\right)_{\operatorname{core}(B)}$. Thus $A \otimes_{\operatorname{core}(B)} A$ is also a direct summand of the $A-C$ bimodule ${ }_{A}\left(A^{n}\right)_{C}$ since $C \subset \operatorname{core}(B)$.

Since $\operatorname{core}(B) \subset B$ the canonical map

$$
A \otimes_{\operatorname{core}(B)} A \rightarrow A \otimes_{B} A
$$

is a surjective morphism of $A-A$-bimodules, in particular of $A-C$ bimodules. Since the category of $A \otimes C^{o p}$-modules is semisimple it follows that $A \otimes_{B} A$ is a direct summand in ${ }_{A}\left(A^{n}\right)_{C}$.

1.6.1. Kernel of a module. Let $A$ be a semisimple Hopf algebra over an algebraically closed field $k$. Then $A$ is also cosemisimple and $S^{2}=\operatorname{Id}$ (see [13]). Let $\Lambda_{A}$ be the idempotent integral of $A$. Denote by $\operatorname{Irr}(A)$ the set of irreducible $A$-characters and let $C(A)$ be the character ring of $A$ with basis $\operatorname{Irr}(A)$. There is an involution "* " on $C(A)$ determined by the antipode.

REMARK 1.4. and If $X \subset C\left(A^{*}\right)$ is closed under multiplication then it generates a subbialgebra of $A$ denoted by $A_{X}[\mathbf{1 5}]$. Moreover if $X$ is also closed under "*" it follows from the same paper that $A_{X}$ is a Hopf subalgebra. Since $A$ is finite dimensional any subbialgebra is a Hopf subalgebra and therefore any subset $X$ closed under multiplication is also closed under "* ".

Let $M$ be an $A$-module with character $\chi$. Define $\operatorname{ker}_{M}$ to be the set of simple subcoalgebras $C$ of $A$ such that $\mathrm{cm}=\varepsilon(c) m$ for all $c \in C$. It can be proven that the set $\operatorname{ker}_{M}$ is closed under multiplication and "*" and therefore from [15] it generates a Hopf subalgebra $A_{M}\left(\right.$ or $\left.A_{\chi}\right)$ of $A[\mathbf{3}]$. One has $A_{M}=\oplus_{C \in \operatorname{ker}_{M}} C$.

REMARK 1.5.

1. $A_{\chi}$ is the largest subbialgebra $B$ of $A$ such that $\chi \downarrow_{B}^{A}=\chi(1) \varepsilon_{B}$. Equivalently, $A_{\chi}$ is the largest subbialgebra $B$ of $A$ such that $A B^{+} A \subset \operatorname{Ann}_{A}(M)$.

2. If $A=k G$ is a group algebra then $A_{\chi}=k[\operatorname{ker} \chi]$ where ker $\chi$ is the kernel of the character $\chi$.

3. It is not known if $A_{\chi}$ is a normal Hopf subalgebra of $A$. In [3] it was proven that $A_{\chi}$ is normal in $A$ if $\chi \in Z\left(A^{*}\right)$. 
4. If $N$ is a submodule or a quotient of $M$ then clearly $A_{M} \subset A_{N}$. (since $A$ is semisimple.)

Notation: If $B$ is a Hopf subalgebra of $A$ then we denote by $\varepsilon \uparrow_{B}^{A}$ the character $\varepsilon_{B} \uparrow_{B}^{A}$.

Proposition 1.6. Suppose $B$ and $C$ are Hopf subalgebras of a finite dimensional semisimple Hopf algebra A. If

$$
{ }_{A}\left(A \otimes_{B} \otimes A\right)_{C} \oplus * \cong_{A} A^{n}{ }_{C}
$$

as $A-C$-bimodules then

$$
C \subset A_{\varepsilon \uparrow_{B}^{A}} .
$$

Proof. As in subsection 1.3.1 it follows that that

$$
\left\langle M \uparrow_{C}^{A} \downarrow_{B}^{A} \uparrow_{B}^{A}, P\right\rangle \leq n\left\langle M \uparrow_{C}^{A}, P\right\rangle
$$

for any simple $C$-module $M$ and any simple $A$-module $P$.

In terms of the characters this can be written as

$$
m_{A}\left(\alpha \uparrow_{C}^{A} \downarrow_{B}^{A} \uparrow_{B}^{A}, \chi\right) \leq n m_{A}\left(\alpha \uparrow_{C}^{A}, \chi\right)
$$

for any irreducible character $\alpha$ of $C$ and any irreducible character $\chi$ of $A$. Here $m_{A}$ is the usual multiplication form on the character ring $C(A)$. Put $\chi=\varepsilon_{A}$, the trivial $A$-character, in the above inequality. Since $m_{A}\left(\alpha \uparrow_{C}^{A}, \varepsilon_{A}\right)$ $=m_{C}\left(\alpha, \varepsilon_{C}\right)$ it follows that $m_{A}\left(\alpha \uparrow_{C}^{A} \downarrow_{B}^{A} \uparrow_{B}^{A}, \varepsilon_{A}\right)=0$ if $\alpha \neq \varepsilon_{C}$. By Frobenius reciprocity this implies that $m_{B}\left(\alpha \uparrow_{C}^{A} \downarrow_{B}^{A}, \varepsilon_{B}\right)=0$ if $\alpha \neq \varepsilon_{C}$. Adding over all irreducible characters $\alpha \in \operatorname{Irr}(C)$ it follows that

$$
m_{B}\left(\left(\sum_{\alpha \in \operatorname{Irr}(C)} \alpha(1) \alpha\right) \uparrow_{C}^{A} \downarrow_{B}^{A}, \varepsilon_{B}\right)=m_{B}\left(\varepsilon \uparrow_{C}^{A} \downarrow_{B}^{A}, \varepsilon_{B}\right)
$$

Since $\sum_{\alpha \in \operatorname{Irr}(C)} \alpha(1) \alpha$ is the regular character of $C$ (see [14]) it follows that $\left(\sum_{\alpha \in \operatorname{Irr}(C)} \alpha(1) \alpha\right) \uparrow_{C}^{A} \downarrow_{B}^{A}$ is the regular character of $B$ multiplied by $\frac{|A|}{|C|}$. Thus $m_{B}\left(\varepsilon \uparrow_{C}^{A} \downarrow_{B}^{A}, \varepsilon_{B}\right)=\frac{|A|}{|B|}$. Frobenius reciprocity implies that $m_{C}\left(\varepsilon \uparrow_{B}^{A} \downarrow_{C}^{A}\right.$, $\left.\varepsilon_{C}\right)=\frac{|A|}{|B|}$. A dimension argument now shows that $\varepsilon \uparrow_{B}^{A} \downarrow_{C}^{A}=\frac{|A|}{|B|} \varepsilon_{C}$ and first item of Remark 1.5 implies that $C \subset A_{\varepsilon \uparrow_{B}^{A}}$.

The above Proposition and Theorem 1.3 suggest the following conjecture:

Conjecture 1. For any Hopf subalgebra $B$ of a semisimple Hopf algebra $A$ one has:

$$
\operatorname{core}(B)=A_{\varepsilon \uparrow \uparrow_{B}^{A}} \text {. }
$$


The next Proposition gives a description of $\operatorname{core}(B)$ in terms of kernels and shows the inclusion $\operatorname{core}(B) \subseteq A_{\varepsilon \uparrow_{B}^{A}}$. In order to prove it we need the following lemmas.

Lemma 1.7. Let $K$ and $L$ be two Hopf subalgebras of a semisimple Hopf algebra $A$. If $\Lambda_{K} \Lambda_{L}=\Lambda_{L}$ then $K \subset L$.

Proof. By Corollary 2.5 of [2] there is a coset decomposition for $A$

$$
A=\oplus_{C / \sim} C L .
$$

where $\sim$ is an equivalence relation on the set of simple subcoalgebras of $A$ given by $C \sim C^{\prime}$ if and only if $C L=C^{\prime} L$. In $[\mathbf{2}]$ this equivalence relation is denoted by $r_{k, L}^{A}$. The equality $\Lambda_{K} \Lambda_{L}=\Lambda_{L}$ shows that any subcoalgebra of $K$ is equivalent to $k 1$ and therefore it is contained in $L$.

Lemma 1.8. Suppose that $B$ is a Hopf subalgebra of a semisimple finite dimensional Hopf algebra. Then $A_{\varepsilon \uparrow_{B}^{A}} \subset B$. Equality holds if and only if $B$ is normal in $A$.

Proof. Let $K=A_{\varepsilon \uparrow_{B}^{A}}$. By the definition of $K$ it follows that $A K^{+}$annihilates $A \otimes_{B} k$. On the other hand $A \otimes_{B} k \cong A / A B^{+}$as $A$-modules and therefore $A K^{+} \subset A B^{+}$. Thus $1-\Lambda_{K} \in A B^{+}$which implies $\Lambda_{K} \Lambda_{B}=\Lambda_{B}$. The above Lemma implies that $K \subset B$. The second statement of the lemma is Corollary 2.5 from $[\mathbf{3}]$.

Proposition 1.9. Suppose that $B$ is a Hopf subalgebra of a semisimple finite dimensional Hopf algebra A. Define inductively

$$
B_{0}=B, \quad B_{r+1}=A_{\varepsilon_{B_{r} A}^{A}} .
$$

Then

$$
B_{1} \supseteq B_{2} \supseteq \cdots \supseteq B_{s} \supseteq B_{s+1} \supseteq \cdots .
$$

If $B_{s}=B_{s+1}$ then $B_{s}=\operatorname{core}(B)$.

Proof. The above proposition implies that $B_{i} \supseteq B_{i+1}$ for any $i$. Since $B$ is finite dimensional there is $s$ such that $B_{s}=B_{s+1}=B_{s+2}=\cdots$. Thus $B_{s}=A_{\varepsilon \uparrow_{B_{s}}^{A}}$ and the above lemma implies that $B$ is normal in $A$. We have to show that $\operatorname{core}(B)=B_{s}$. Suppose that $K$ is normal in $A$ and that $K \subseteq B$. It is enough to show $K \subseteq B_{s}$. Clearly $K \subseteq B_{0}$. If $K \subseteq B_{i}$ then there is a canonical surjection of $A$-modules $A / A K^{+} \rightarrow A / A B_{i}^{+}$. Thus $A_{\varepsilon \uparrow_{K}^{A}} \subset A_{\varepsilon_{\varepsilon_{B}}^{A} B_{i}}$ by the last item of Remark 1.5. On the other hand $A_{\varepsilon \uparrow_{K}^{A}}=K$ since $K$ is normal. Therefore $K \subseteq B_{i+1}$. 
1.6.2. The correspondent of conjugate Hopf subalgebras. Let $A$ be a semisimple Hopf algebra over an algebraically closed field $k$ and let $\widehat{A^{*}}$ be the set of simple subcoalgebras of $A$. Since $A$ is cosemisimple note that $\widehat{A^{*}}$ can be identified with $\operatorname{Irr}\left(A^{*}\right)[\mathbf{1 2}]$. Let $B$ be a Hopf subalgebra of $A$ and $C$ a simple subcoalgebra of $A$. Define

$$
X_{C_{B}}=\left\{D \in \widehat{A^{*}} \mid d c \Lambda_{B}=\varepsilon(d) c \Lambda_{B} \text { for all } c \in C, d \in D\right\}
$$

Proposition 1.10. The set $X_{C_{B}}$ is closed under multiplication and "*" and it generates a Hopf subalgebra ${ }^{C} B$ of $A$.

Proof. By Remark 1.4 it is enough to show that the above set is closed under multiplication. Suppose that $D$ and $D^{\prime}$ are subcoalgebras in $X_{C_{B}}$. If $E$ is a simple subcoalgebra of $D D^{\prime}$ then any $e \in E$ can be written as $\sum_{i=1}^{s} d_{i} d_{i}^{\prime}$ with $d_{i} \in D$ and $d_{i}^{\prime} \in D^{\prime}$. Then $e c \Lambda_{B}=\varepsilon(e) c \Lambda_{B}$ which show that $E \in X_{C_{B}}$.

Notation: ${ }^{C} B$ will also be denoted with ${ }^{c} B$ if $c$ is the irreducible character of $A^{*}$ corresponding to $C$.

EXAMPLE 1.11. Let $A=k G$ and $B=k N$ where $N$ is a subgroup of $G$. The simple subcoalgebras of $A$ are $k g$ with $g \in G$ and $\Lambda_{B}=\frac{1}{|N|} \sum_{n \in N} n$. Then ${ }^{g} B=g B g^{-1}$ for all $g \in G$. Indeed $X_{g_{B}}=\left\{h \in G \mid h g \Lambda_{B}=g \Lambda_{B}\right\}=\{h \in$ $G \mid h g N=g N\}=g N g^{-1}$

Proposition 1.12. Let $B$ be a Hopf subalgebra of $A$ and $g \in G(A)$ be a grouplike element of $A$. Then ${ }^{g} B=g B g^{-1}$.

Proof. First note that ${ }^{1} B=B$. Clearly $B \subset{ }^{1} B$. On the other hand the definition of ${ }^{1} B$ implies that $\Lambda_{1_{B}} \Lambda_{B}=\Lambda_{B}$. Then Lemma 1.7 implies ${ }^{1} B \subset B$.

Let now $C$ be a simple subcoalgebra of ${ }^{g} B$. Then $c g \Lambda_{B}=\varepsilon(c) g \Lambda_{B}$ for all $c \in C$. Thus $g^{-1} c g \Lambda_{B}=\varepsilon(c) \Lambda_{B}$ which shows that $g^{-1} C g \subset{ }^{1} B=B$. Therefore $C \subset g B g^{-1}$ which shows that ${ }^{g} B \subset g B g^{-1}$. A direct computations shows that $g B g^{-1} \subset^{g} B$. Thus ${ }^{g} B=g B g^{-1}$.

Proposition 1.13. Let $B$ be a Hopf subalgebra of $A$. Then

$$
A_{\varepsilon \uparrow_{B}^{A}}=\cap_{C \in \widehat{A}^{*}}{ }^{C} B .
$$

Proof. Recall the coset decomposition

$$
A=\oplus_{C / \sim} C B .
$$

form Corollary 2.5 of [2]. If $k$ is the trivial $B$-module then

$$
k \uparrow_{B}^{A}=\oplus_{C / \sim} C B \otimes_{B} k .
$$


From the definition of ${ }^{C} B$ it follows that $C B \otimes_{B} k$ is trivial as left ${ }^{C} B$-module. Therefore $\cap_{C \in \widehat{A}^{*}}{ }^{C} B \subset A_{\varepsilon \uparrow_{B}^{A}}$.

Note that $k \uparrow_{B}^{A}=A \otimes_{B} k \cong A \Lambda_{B}$ as left $A$-modules via $a \Lambda_{B} \mapsto a \otimes_{B} \Lambda_{B}$. The decomposition 15 implies that $C B \otimes_{B} k \cong C \Lambda_{B}$ under the above isomorphism. Any simple subcoalgebra of $A_{\varepsilon \uparrow_{B}^{A}}$ acts trivially on $k \uparrow_{B}^{A}$ and therefore on each $C B \otimes_{B} k$. This implies that any such coalgebra is contained in ${ }^{C} B$. Thus $A_{\varepsilon \uparrow_{B}^{A}} \subset^{C} B$ for any simple coalgebra $C \in \widehat{A^{*}}$.

Corollary 1.14. Let $B$ be a Hopf subalgebra of $A$. Then $B$ is a normal Hopf subalgebra if and only if

$$
B=\cap_{C \in \widehat{A}^{*}}^{C} B .
$$

Proof. Since $A_{\varepsilon \uparrow_{B}^{A}}=\cap_{C \in \widehat{A^{*}}}^{C} B$ this is Corollary 2.5 of $[\mathbf{3}]$.

REMARK 1.15.

1. Theorem 1.9 implies that $\operatorname{core}(B) \subset A_{\varepsilon \uparrow_{B}^{A}}=\cap_{C \in \widehat{A^{*}}}^{C} B$. This can also be seen directly as follows. Fix $C \in \widehat{A^{*}}$. For any $x \in \operatorname{core}(B)$ and $c \in$ $C$ one has that $x c \Lambda_{B}=c_{1}\left(S\left(c_{2}\right) x c_{3}\right) \Lambda_{B}=c_{1} \varepsilon(x) \varepsilon\left(c_{2}\right) \Lambda_{B}=\varepsilon(x) c \Lambda_{B}$ since $\operatorname{core}(B)$ is normal in $A$. Thus $\operatorname{core}(B) \subset^{C} B$.

2. If $A_{\chi}$ is normal Hopf algebra for any $\chi \in \operatorname{Irr}(A)$ then Proposition 1.9 implies the above conjecture on the core of a Hopf subalgebra.

\section{Depth three Frobenius extension}

A Frobenius extension $A \mid B$ is defined to be depth three if the following tower of subalgebras in the endomorphism ring $E=$ End $A_{B}$ is right or left depth-3: via the algebra monomorphism, left multiplication $\lambda: A \hookrightarrow E$ given by $\lambda(a)(x)=a x(x, a \in A)$ we obtain the (ascending) tower, $\lambda(B) \subseteq \lambda(A) \subseteq$ $E$. By [11, Theorem 3.1] the given tower is left $\mathrm{d}-3$ if and only if the tower is right $d-3$.

The definitions and first properties of depth two and three extensions are introduced in detail in $[\mathbf{1 1}]$. There it is determined that a tower of three group algebras corresponding to the subgroup chain $G \geq H \geq K$ is depth three if the normal closure $K^{G}$ (of $K$ in $G$ ) is contained in $H$. In [10] it is shown that, with $k=\mathbb{C}$ and $G$ a finite group, the group algebra $A$ of $G$ is depth two over subgroup algebra $B$ of $H$ if and only if $H$ is a normal subgroup of $G$. This normality result for depth two subalgebras is extended to semisimple Hopf algebras over an algebraically closed field of characteristic zero in [4].

The following is a characterization of depth three for a separable, Frobenius extension in terms of the more familiar depth two property. The following is true more generally for QF-extensions [5, Theorem 3.8]. 
Theorem 2.1. Suppose $A \mid B$ is a separable extension and Frobenius extension. Let $E$ denote End $A_{B}$ and $\lambda: A \hookrightarrow E$ be understood as the extension $E \mid A$. The $A \mid B$ is depth three if and only if the composite extension $E \mid B$ is depth two.

Sketch of Proof. $(\Rightarrow)$ This direction does not apply separability. By the Frobenius extension property, we noted above that $E \cong A \otimes_{B} A$ as $(A, A)$ bimodules. Then $E \otimes_{A} E \otimes_{A} E \cong E \otimes_{B} E$ as natural ( $\left.E, E\right)$-bimodules. By definition of right D3 extension, $E \otimes_{A} E$ is isomorphic to direct summand of $E^{n}$ as natural $(E, B)$-bimodules for some $n \in \mathbb{N}$, whence $E \otimes_{A} E \otimes_{A} E \cong$ $E \otimes_{B} E$ is $(E, B)$-isomorphic to a direct summand of $E \otimes_{A} E^{n}$, which in turn is isomorphic to a direct summand of $E^{n^{2}}$ by the right D3 property. Hence $E \mid B$ is right D2, since $E \otimes_{B} E \oplus * \cong E^{n^{2}}$ as natural $(E, B)$-bimodules.

$(\Leftarrow)$ There is a split $(E, B)$-epimorphism from $E^{n} \rightarrow E \otimes_{B} E$ for some $n \in$ $N$. In addition, there is a split $(E, E)$-epimorphism from $E \otimes_{B} E \rightarrow E \otimes_{A} E$ by the separability property of the extension $A \mid B$. Composing the two split epis we obtain a split epi $E^{n} \rightarrow E \otimes_{A} E$ showing $A \mid B$ is right D3.

Proposition 2.2. Let $M$ be the inclusion matrix of a subalgebra pair of semisimple complex algebras $B \subseteq A$, and $\mathcal{S}=M M^{t}$. The symmetric matrix $\mathcal{S}$ satisfies

$$
\mathcal{S}^{3} \leq n \mathcal{S}
$$

for some positive integer $n$ if and only if $B$ is a depth three subalgebra of $A$.

Proof. Let $M_{m}(\mathbb{C})=\operatorname{End}_{\mathbb{C}} A=\mathcal{E}$ where $m=\operatorname{dim} A$, which contains both $A$ and $B$ via left regular representation. It is shown in $[\mathbf{7}, 2.3 .5]$ that the centralizers $\mathcal{E}^{A} \subseteq \mathcal{E}^{B}$ have transpose inclusion matrix; i.e. inclusion matrix of $A \hookrightarrow$ End $A_{B}$ via $a \mapsto \lambda_{a}$ is $M^{t}$. It is not hard to show from transitivity of induction that matrix multiplication yields new inclusion matrix of two successive subalgebra pairs. Hence, inclusion matrix of $B \hookrightarrow E$ via $b \mapsto \lambda_{b}$ $(b \in B)$ is given by $M M^{t}$.

The algebra $A$ is separable, whence separable extension over $B$. The extension $A \supseteq B$ is a split Frobenius extension by application of [7, Goodman-De la Harpe-Jones, ch. 2], very faithful conditional expectations. Then $A_{B}$ is a progenerator since $B$ is semisimple and $B \hookrightarrow A$ is split $B$-module monic, so $E$ and $B$ are Morita equivalent semisimple algebras. By the theorem above, $B \subseteq A$ is depth three iff $B \hookrightarrow E$ is depth two, and we may apply Proposition 1.2 to the composite inclusion matrix $\mathcal{S}=M M^{t}$.

In general for any subgroup $H$ in finite group $G$ with inclusion matrix $M$, if the irreducible characters of $H$ are given by $\left\{\psi_{1}, \ldots, \psi_{r}\right\}=\operatorname{Irr}(H)$, note that the matrix $\mathcal{S}=M M^{t}$ is given by

$$
\mathcal{S}=\left(\begin{array}{ccc}
\left\langle\psi_{1}^{G} \mid \psi_{1}^{G}\right\rangle & \ldots & \left\langle\psi_{1}^{G} \mid \psi_{r}^{G}\right\rangle \\
\ldots & \ldots & \ldots \\
\left\langle\psi_{r}^{G} \mid \psi_{1}^{G}\right\rangle & \ldots & \left\langle\psi_{r}^{G} \mid \psi_{r}^{G}\right\rangle
\end{array}\right)
$$


For example, we revisit the inclusion $S_{2}<S_{3}$ analyzed above. Note that

$$
\mathcal{S}=M M^{t}=\left(\begin{array}{ll}
2 & 1 \\
1 & 2
\end{array}\right) .
$$

Since $\mathcal{S}$ is strictly positive (i.e. has only positive whole number entries), it is clear that there is positive integer $n$ such that $\mathcal{S}^{3} \leq n \mathcal{S}$.

The notation in the proposition above with finite dimensional complex group algebras $B=\mathbb{C}[H]$ and $A=\mathbb{C}[G]$ is continued in the next corollary:

Corollary 2.3. The subgroup $H$ is depth three in $G$ if symmetric matrix $\mathcal{S}$ is strictly positive.

Another example: the standard inclusion of full permutation group algebras $B=\mathbb{C}\left[S_{3}\right] \hookrightarrow \mathbb{C}\left[S_{4}\right]=A$ has inclusion matrix (computed from character tables in e.g. [6]) and symmetric matrix:

$$
M=\left(\begin{array}{lllll}
1 & 0 & 0 & 1 & 0 \\
0 & 1 & 0 & 0 & 1 \\
0 & 0 & 1 & 1 & 1
\end{array}\right) \quad \mathcal{S}=\left(\begin{array}{lll}
2 & 0 & 1 \\
0 & 2 & 1 \\
1 & 1 & 3
\end{array}\right) \quad S^{3}=\left(\begin{array}{ccc}
15 & 7 & 21 \\
7 & 15 & 21 \\
21 & 21 & 43
\end{array}\right)
$$

It is clear that there is no positive integer $n$ for which $\mathcal{S}^{3} \leq n \mathcal{S}$, since $\mathcal{S}$ has zero entries but $\mathcal{S}^{3}$ is strictly positive. We conclude that $S_{3}$ is not a depth three subgroup of $S_{4}$. (Using the next theorem one computes that $S_{3}$ is a depth five subgroup of $S_{4}$.)

2.1. Higher depth. Recall from [11] that depth $n>2$ is defined as follows. Begin with a Frobenius extension (or QF extension [5]) $B=A_{-1} \subseteq$ $A=A_{0}$. Let $A_{1}=$ End $A_{B}$ and inductively $A_{n}=\operatorname{End}\left(A_{n-1}\right)_{A_{n-2}}$. By the Frobenius hypothesis and its endomorphism ring theorem, $A_{n} \cong A \otimes_{B} \cdots \otimes_{B}$ $A(n+1$ times $A)$. Embedding $A_{n} \hookrightarrow A_{n+1}$ via left regular representation $\lambda$, we obtain a Jones tower of algebras,

$$
B \hookrightarrow A \hookrightarrow A_{1} \hookrightarrow \cdots \hookrightarrow A_{n} \hookrightarrow A_{n+1} \hookrightarrow \cdots
$$

The subalgebra $B$ in $A$ is depth $n$ if $A_{n-2} \supseteq A_{n-3} \supseteq B$ is a depth-3 tower defined above; infinite depth if there is no such positive integer $n$. Of course, this agrees with the definition of depth three subalgebra above. If $B$ and $A$ are semisimple complex algebras, $A \supseteq B$ becomes a split, separable Frobenius extension via the construction of a very faithful conditional expection [7]. This type of extension has an endomorphism ring theorem [9], and enjoys transitivity, so that all extensions in this Jones tower are split, separable Frobenius extensions, and all algebras are semisimple by Morita's theorem (or Serre's theorem on global dimension). Indeed, all the odd $A_{n}$ 's are Morita equivalent to $B$, while all the even $A_{n}$ 's are Morita equivalent to $A$. The proof of the lemma below is similar to that of Prop. 1.2 and therefore omitted. (One notes that $\operatorname{Ind}_{C}^{A} \cong \operatorname{Ind}_{B}^{A} \operatorname{Ind}_{C}^{B}$ is given by the rows of matrix $N M$.) 
Lemma 2.4. Suppose $C \subseteq B \subseteq A$ is a tower of semisimple algebras with inclusion matrices $N$ and $M$ respectively. Then the tower is depth-3 if and only if there is a positive integer $n$ such that

$$
N M M^{t} M \leq n N M
$$

Notice that Prop. 1.2 follows from letting $B=C$ and $N$ equal the identity matrix of rank $\operatorname{dim} Z(B)$. Conversely, if $A \supseteq B$ is depth two, and $C$ any subalgebra of $B$, then the lemma follows in this special case from Prop. 1.2 by multiplying the inequality there from the left by the inclusion matrix $N$ of $C \subseteq B$.

Let $n, m$ and $q$ denote positive integers below.

Theorem 2.5. Suppose $B \subseteq A$ is a subalgebra pair of semisimple algebras. Let $M$ be the inclusion matrix and $\mathcal{S}=M M^{t}$. If $n=2 m+1$ then $A \supseteq B$ is depth $n$ if and only if $\mathcal{S}^{m+1} \leq q \mathcal{S}^{m}$ for some $q$. If $n=2 m$, then $A \supseteq B$ is depth $n$ if and only if $\mathcal{S}^{m} M \leq q \mathcal{S}^{m-1} M$ for some $q$.

Proof. The proof follows from noting that if $M$ is the inclusion matrix of $B \subseteq A$, then $M^{t}$ is the inclusion matrix of $A \hookrightarrow A_{1}$, and $\mathcal{S}$ is the inclusion matrix of their composite $B \hookrightarrow A_{1}$. The proof now follows from applying the last lemma to the depth-3 tower $B \hookrightarrow A_{n-3} \hookrightarrow A_{n-2}$ in the even and odd case.

It is worth emphasizing that a depth $n$ algebra extension is also depth $n+1$ (so one might denote this as depth $\geq n$ ); in the special case of the theorem, this is seen by multiplying the given inequality from the right by the inclusion matrix $M$ or $M^{t}$. Of course one should strive to use the least depth to one's knowledge. Let $m$ be a positive integer and $G$ a finite group in the next result on subgroups of finite depth.

Corollary 2.6. Suppose $H<G$ is a subgroup with symmetric matrix $\mathcal{S}$. If $\mathcal{S}^{m}$ is a strictly positive matrix, then $H$ is a subgroup of depth $2 m+1$ in $G$.

Proof. Applying the theorem we see $\mathcal{S}^{m+1} \leq q \mathcal{S}^{m}$ for some positive integer $q$ since $\mathcal{S}^{m}$ is a strictly positive matrix.

For example, while $S_{3}<S_{4}$ is not D3 subgroup, we note that $\mathcal{S}^{2}$ is already strictly positive order 3 matrix, whence it is a depth five subgroup (and it may be checked that it is not depth four).

As another example of a more cautionary note, the symmetries of a square $D_{4}$ in $S_{4}$ has zero entries in all powers of its order 5 matrix $\mathcal{S}=M M^{t}$. However, one computes that $\mathcal{S}^{2} M \leq 4 \mathcal{S} M$, so that $D_{4}$ is a depth four subgroup of $S_{4}$ according to Theorem 2.5.

In a forthcoming paper it will be shown that after a permutation of the indices, the matrix $S$ can be written as a sum of diagonal blocks. Moreover there is $p>0$ such that the $p$-power of each diagonal block is a positive 
matrix. Applying Theorem 2.5 this implies that the extension $B \subseteq A$ is of depth at least $2 p+1$; in other words, all semisimple subalgebra pairs are of finite depth.

2.2. Simplified condition for depth three. Again let $A \supseteq B$ be an algebra extension. In case $A_{B}$ is a generator, such as when the extension is free or right split, there is a particularly simplified condition for when a Frobenius extension is depth three.

TheOrem 2.7. Suppose $A \supseteq B$ is a Frobenius extension where the natural module $A_{B}$ is a generator. Then $A \supseteq B$ is depth three if and only if there is a $B$-B-bimodule $P$ and positive integer $n$ such that

$$
{ }_{B} A \otimes_{B} A_{B} \oplus P \cong{ }_{B} A_{B}{ }^{n}
$$

Proof. $(\Rightarrow)$ Let $E=$ End $A_{B}$. By the Frobenius extension hypothesis on $A \supseteq B$, as $E$ - $A$-bimodules $E \cong A \otimes_{B} A$ via the mapping in subsection 1.1. Recall that $A \supseteq B$ is depth three if $B \subseteq A \hookrightarrow E$ is depth three tower, i.e. ${ }_{E} E \otimes_{A} E_{B} \oplus Q \cong{ }_{E} E_{B}^{n}$ for some $E$-B-bimodule $Q$ and positive integer $n$. Then by substitution

$$
{ }_{E} A \otimes_{B} A \otimes_{B} A_{B} \oplus Q \cong{ }_{E} A \otimes_{B} A_{B}^{n} .
$$

But $A_{B}$ is a progenerator by hypothesis, whence $B$ and $E$ are Morita equivalent algebras. The context bimodule are ${ }_{B} \operatorname{Hom}\left(A_{B}, B_{B}\right)_{E}$ (the right $B$ dual of $A$ denoted by $\left.\left(A_{B}\right)^{*}\right)$ and ${ }_{E} A_{B}$ with $B$ - $B$-bimodule isomorphism $\operatorname{Hom}\left(A_{B}, B_{B}\right) \otimes_{E} A \stackrel{\cong}{\longrightarrow} B$ given by evaluation. Now tensor all components of eq. (21) by ${ }_{B}\left(A_{B}\right)^{*} \otimes_{E}-$ and cancel $B \otimes_{B}$ to obtain eq. (20), where of course $P=\left(A_{B}\right)^{*} \otimes_{E} Q$.

$(\Leftarrow)$ Tensor all components of eq. $(20)$ from the left by the natural bimodule ${ }_{E} A_{B}$ given by $f \cdot a \cdot b=f(a) b$, obtain eq. (21), and reverse the argument above it. This direction of proof does not make use of generator hypothesis.

REMARK 2.8. Since ${ }_{B} A_{B} \oplus \Omega \cong{ }_{B} A \otimes_{B} A_{B}$ is always the case for some $B$ - $B$-bimodule $\Omega$, the $B$ - $B$-bimodules $A \otimes_{B} A$ and $A$ are similar or $\mathrm{H}$ equivalent under the conditions of the theorem: thus their endomorphism algebras are Morita equivalent. By Theorem 2.1, left multiplication $B \hookrightarrow E$ is depth two. There is a general Galois theory of depth two extensions which in this case specializes to total algebra End ${ }_{B} A \otimes_{B} A_{B}$ and base algebra $E^{B} \cong$ End $_{B} A_{B}$ as parts of a bialgebroid. It is interesting to note that base and total algebras in this case are Morita equivalent.

Let $\operatorname{Res}=\operatorname{Res}_{H}^{G}$ denote restriction of $G$-modules to $H$-modules in the corollary below, and $\operatorname{Ind}=\operatorname{Ind}_{H}^{G}$ denote induction of $H$-modules to $G$-modules. 
Corollary 2.9. A subgroup $H$ of a finite group $G$ is depth three if

$$
\langle\operatorname{ResIndResInd} \psi \mid \chi\rangle \leq n\langle\operatorname{ResInd} \psi \mid \chi\rangle
$$

for all irreducible characters $\psi, \chi$ of $H$.

Proof. Note that the corresponding complex group algebras $A \supseteq B$ satisfy the conditions of the theorem. One arrives at the condition on inner products of characters by tensoring a simple $B$-module $V$ by the components in eq. (20). Of course, whatever simple $B$-module components of ${ }_{B} A \otimes_{B} A \otimes$ ${ }_{B} V$ has, also ${ }_{B} A^{n} \otimes_{B} V$ has.

For example, from the character tables of the permutation groups $S_{4}$ and $S_{5}$ [6] we compute the induction-restriction table by restricting irreducible characters on $S_{5}$, given below in matrix form (with first column and row corresponding to trivial characters):

$$
M=\left(\begin{array}{lllllll}
1 & 0 & 1 & 0 & 0 & 0 & 0 \\
0 & 1 & 0 & 1 & 0 & 0 & 0 \\
0 & 0 & 0 & 0 & 0 & 1 & 1 \\
0 & 0 & 1 & 0 & 1 & 1 & 0 \\
0 & 0 & 0 & 1 & 1 & 0 & 1
\end{array}\right)
$$

Let $\eta_{i} \in \operatorname{Irr}(H)$ and $\chi_{i} \in \operatorname{Irr}(G)(i=1, \ldots, 5)$. Then from row $1, \eta_{1}^{G}=$ $\chi_{1}+\chi_{3}, \eta_{1}^{G} \downarrow_{H}=2 \eta_{1}+\eta_{4}$ and finally $\eta_{1}^{G} \downarrow_{H} \uparrow^{G} \downarrow_{H}=5 \eta_{1}+\eta_{3}+5 \eta_{4}+\eta_{5}$. Note $\left\langle\eta_{1}^{G} \downarrow_{H} \uparrow^{G} \downarrow_{H} \mid \eta_{3}\right\rangle=1 \not \leq n\left\langle\eta_{1}^{G} \downarrow_{H} \mid \eta_{3}\right\rangle=0$ for all positive integers $n$, whence $S_{4}$ is not a D3 subgroup in $S_{5}$.

Computing the $5 \times 5$ matrix $\mathcal{S}=M M^{t}$, we may compute that the matrix $\mathcal{S}^{3}$ and $\mathcal{S}^{4}$ are strictly positive, so that $S_{4}$ is a depth seven subgroup in $S_{5}$ by Theorem 2.5 and its corollary. (Observing the pattern, we might conjecture at this point that the canonical subgroup $S_{n}<S_{n+1}$ has depth $2 n-1$.)

2.3. Depth three quasi-bases. The condition (20) for a depth three extension has an interpretation in terms of split epis, including the canonical split epis of a product. This should give us depth three condition in terms of quasi-bases somewhat similar to dual bases for projective modules. Meanwhile the Frobenius hypothesis on extension $A \supseteq B$ is needed to reduce the quasi-bases to simplest terms. Suppose $F$ is a Frobenius homomorphism $A \rightarrow B$ with dual bases $\left\{x_{i}\right\}$ and $\left\{y_{i}\right\}$ in $A$.

Theorem 2.10. Suppose $A \supseteq B$ is a Frobenius extension where $A_{B}$ is a generator. Then $A \supseteq B$ is a depth three extension if and only if there are elements $u_{i}, t_{i} \in\left(A \otimes_{B} A \otimes_{B} A\right)^{B}$ such that for all $x, y \in A$,

$$
x \otimes_{B} y=\sum_{i=1}^{n} t_{i}^{1} \otimes_{B} t_{i}^{2} F\left(t_{i}^{3} u_{i}^{1} F\left(u_{i}^{2} F\left(u_{i}^{3} x\right) y\right)\right)
$$

where $u=u^{1} \otimes u^{2} \otimes u^{3}$ is Sweedler notation that suppresses a possible summation over simple tensors. 
Proof. $(\Rightarrow)$ First note from eq. (20) that there are mappings $f_{i} \in \operatorname{Hom}\left({ }_{B} A_{B},{ }_{B} A \otimes_{B} A_{B}\right)$ and $g_{i} \in \operatorname{Hom}\left({ }_{B} A \otimes_{B} A_{B},{ }_{B} A_{B}\right)$ such that

$$
\sum_{i=1}^{n} f_{i} \circ g_{i}=\operatorname{id}_{A \otimes_{B} A}
$$

Next recall that for any $B$-module $M$, CoInd $M \cong \operatorname{Ind} M$ for a Frobenius extension $A$ over $B$ [9]; I.e., there is a natural $A$-module isomorphism Hom $\left(A_{B}, M_{B}\right) \cong M \otimes_{B} A$ via $f \mapsto \sum f\left(x_{i}\right) \otimes y_{i}$ with inverse $m \otimes a \mapsto$ $m F(a-)$. Applied to $M=A \otimes_{B} A$, this restricts to $\operatorname{Hom}\left({ }_{B} A_{B},{ }_{B} A \otimes_{B} A_{B}\right) \cong$ $\left(A \otimes_{B} A \otimes_{B} A\right)^{B}$ via $f \mapsto \sum_{i} f\left(x_{i}\right) \otimes y_{i}$ with inverse

$$
t \longmapsto t^{1} \otimes t^{2} F\left(t^{3}-\right) \text {. }
$$

Next apply the hom-tensor relation and the Frobenius isomorphism between endomorphism ring and tensor-square of extension:

$$
\begin{aligned}
\operatorname{Hom}\left({ }_{B} A \otimes_{B} A_{B},{ }_{B} A_{B}\right) & \cong \operatorname{Hom}\left(A_{B}, E_{B}\right)^{B} \\
& \cong \operatorname{Hom}\left({ }_{B} A_{B},{ }_{B} A \otimes_{B} A_{B}\right) \cong\left(A \otimes_{B} A \otimes_{B} A\right)^{B} .
\end{aligned}
$$

Following the isomorphisms, the forward composite mapping is given by $g \mapsto \sum_{i, j} g\left(x_{i} \otimes x_{j}\right) \otimes y_{j} \otimes y_{i}$ with inverse given by

$$
u \longmapsto\left(x \otimes y \mapsto u^{1} F\left(u^{2} F\left(u^{3} x\right) y\right)\right)
$$

for all $u \in\left(A \otimes_{B} A \otimes_{B} A\right)^{B}, x, y \in A$.

Now suppose the mappings we begin with $f_{i} \mapsto t_{i}$ and $g_{i} \mapsto u_{i}$ in $\left(A \otimes_{B}\right.$ $\left.A \otimes_{B} A\right)^{B}$ via isomorphisms displayed above. Then eq. (23) results.

$(\Leftarrow)$ Define a split $B$ - $B$-bimodule epimorphism $A^{n} \rightarrow A \otimes_{B} A$ by $\left(a_{1}, \ldots\right.$, $\left.a_{n}\right) \mapsto \sum_{i=1}^{n} t_{i}^{1} \otimes t_{i}^{2} F\left(t_{i}^{3} a_{i}\right)$ with section $A \otimes_{B} A \rightarrow A^{n}$ given by $x \otimes y \mapsto$ $\left(u_{i}^{1} F\left(u_{i}^{2} F\left(u_{i}^{3} x\right) y\right)\right)_{i=1, \ldots, n}$.

For example, a left depth two quasi-bases $t_{i} \in\left(A \otimes_{B} A\right)^{B}$ and $\beta_{i} \in$ End ${ }_{B} A_{B}$ for $A \supseteq B$ satisfy $x \otimes y=\sum_{i=1}^{n} t_{i} \beta_{i}(x) y$ for all $x, y \in A$. If $A$ is Frobenius extension of $B$, then End $_{B} A_{B} \cong\left(A \otimes_{B} A\right)^{B}$ via $\alpha \mapsto \sum_{i} \alpha\left(x_{i}\right) \otimes y_{i}$ with inverse $t \mapsto t^{1} F\left(t^{2}-\right)$. Let $u_{i} \in\left(A \otimes_{B} A\right)^{B}$ satisfy $u_{i}^{1} F\left(u_{i}^{2}-\right)=\beta_{i}$. Then

$$
\left\{\sum_{j} t_{i}^{1} \otimes_{B} t_{i}^{2} x_{j} \otimes_{B} y_{j}\right\}_{i=1, \ldots, n}\left\{\sum_{j} x_{j} \otimes_{B} y_{j} u_{i}^{1} \otimes_{B} u_{i}^{2}\right\}_{i=1, \ldots, n}
$$

are D3 quasi-bases, because

$$
\begin{aligned}
\sum_{i, j, k} t_{i}^{1} \otimes t_{i}^{2} x_{j} F\left(y_{j} x_{k} F\left(y_{k} u_{i}^{1} F\left(u_{i}^{2} x\right) y\right)\right) & =\sum_{i, k} t_{i}^{1} \otimes t_{i}^{2} x_{k} F\left(y_{k} u_{i}^{1} F\left(u_{i}^{2} x\right) y\right) \\
& =\sum_{i} t_{i}^{1} \otimes t_{i}^{2} u_{i}^{1} F\left(u_{i}^{2} x\right) y=x \otimes y
\end{aligned}
$$




\section{Hall subgroup in Frobenius group is depth three}

A Frobenius group is a finite group $G$ with nontrivial normal subgroup $M$ (called the Frobenius kernel) which contains the centralizer of each of its nonzero elements: $C_{G}(\{x\}) \subseteq M$ for each $x \in M^{*}[\mathbf{8}, \mathbf{1 7}]$. This is equivalent to $G$ having a Hall subgroup, or Frobenius complement, $H$ such that $G=$ $M H, M \cap H=\{e\}, H \cap H^{x}=\{e\}$ where $H^{x}=x^{-1} H x$ for any $x \in G-H$; in addition, $M=G-\cup_{x \in G} x^{-1} H^{*} x$. The Hall subgroup $H$ is not normal in $G$ (and therefore not depth two in the terms of this paper). We will see below that $H<G$ represents a nontrivial class of examples of depth three subgroup.

For example, the permutation group $S_{3}$ is a Frobenius group with kernel $M=\langle(123)\rangle$ and $H=S_{2}=\langle(12)\rangle$ or either of the two subgroups $\langle(23)\rangle$ or $\langle(13)\rangle$ are Hall subgroups.

Theorem 3.1. Let $G$ be a Frobenius group with Hall subgroup H. Then $H$ is depth three subgroup of $G$.

Proof. From the defining condition (22), we easily find a positive integer $n$ if $\langle\operatorname{ResInd} \psi \mid \chi\rangle>0$ for all irreducible characters $\psi, \chi$ of $H$. We compute using Mackey subgroup theorem [8, p. 74] and Frobenius reciprocity, where $T$ denotes a set of $n$ double coset representative $\left\{e=g_{1}, g_{2}, \ldots, g_{n}\right\}$ :

$$
\left\langle\psi^{G} \downarrow_{H} \mid \chi\right\rangle=\sum_{t \in T}\left\langle\left(\psi^{t} \downarrow_{H^{t} \cap H}\right) \uparrow^{H}, \chi\right\rangle=\sum_{t \in T}\left\langle\psi^{t} \downarrow_{H^{t} \cap H} \mid \chi \downarrow_{H^{t} \cap H}\right\rangle \geq n-1
$$

since $H^{t} \cap H=\{e\}$ for each $t \neq g_{1}$. Indeed it is easy to check that

$$
\left\langle\psi^{G} \downarrow_{H} \mid \chi\right\rangle=(n-1)(\operatorname{deg} \psi)(\operatorname{deg} \chi)
$$

if $\psi \neq \chi$ and equals $1+(n-1)(\operatorname{deg} \psi)^{2}$ if $\chi=\psi$.

For example the subgroup $S_{2}$ in $S_{3}$ has two double coset reprentatives, both irreducible characters are linear, and the values $\left\langle\psi^{G} \downarrow_{H} \mid \chi\right\rangle>=$

$\left\langle\psi^{G} \mid \chi^{G}\right\rangle$ are 1 on the off-diagonal and 2 on the diagonal, the coefficients of the matrix $\mathcal{S}$ in eq. (18). The proof of the theorem also follows from eq. (17), Corollary 2.3 and Mackey's theorem.

\section{Acknowledgments}

The second author is grateful to David Harbater and Gestur Olafsson for discussions related to this paper. The first author was supported by the strategic grant POSDRU/89/1.5/S/58852, Project "Postdoctoral programme for training scientific researchers" cofinanced by the European Social Found within the Sectorial Operational Program Human Resources Development 2007-2013. 


\section{References}

[1] J. L. Alperin with R. B. Bell, Groups and Representations, GTM 162, Springer, New York, 1995.

[2] S. Burciu, Coset decomposition for semisimple Hopf Algebras, Comm. Alg., 37, 10, (2009), 3573-3585.

[3] S. Burciu, Normal Hopf subalgebras of a semisimple Hopf algebra, Proc. A. M. S., 12 (2009), 3969-3979.

[4] S. Burciu and L. Kadison, Depth two Hopf subalgebras of a semisimple Hopf algebra J. Algebra, 322, (2009), 162-176.

[5] F. Castano Iglesias and L. Kadison, Similarity, codepth two bicomodules and QF bimodules, preprint arXiv:0712.4362.

[6] W. Fulton and J. Harris, Representation Theory, GTM 129, Springer, 1991.

[7] F. Goodman, P. de la Harpe, and V.F.R. Jones, Coxeter Graphs and Towers of Algebras, M.S.R.I. Publ. 14, Springer, Heidelberg, 1989.

[8] I. M. Isaacs, Character Theory of Finite Groups, Dover, New York, 1976.

[9] L. Kadison, New Examples of Frobenius Extensions, Univ. Lect. Ser. 14, A.M.S., 1999.

[10] L. Kadison and B. Külshammer, Depth two, normality and a trace ideal condition for Frobenius extensions, Comm. Alg. 34 (2006), 3103-3122.

[11] L. Kadison, Finite depth and Jacobson-Bourbaki correspondence, J. Pure \& Applied Alg. 212 (2008), 1822-1839.

[12] R. G. Larson, Characters of Hopf algebras, J. Algebra 17 (1971), 352-368.

[13] R. G. Larson, and D. E. Radford, Finite dimensional cosemisimple Hopf Algebras in characteristic zero are semisimple, J. Algebra 117 (1988), 267-289.

[14] S. Montgomery, Hopf Algebras and Their Actions on Rings, CBMS Regional Conf. Series in Math. Vol. 82, AMS, Providence, 1993.

[15] W. D. Nichols and M. B. Richmond, The Grothendieck group of a Hopf algebra, I, Comm. Alg. 26 (1998), 1081-1095.

[16] M. Rieffel, Category theory and quantum field theory, in: Noncommutative Rings(Berkeley, CA, 1989), 115-129, Math. Sci. Res. Inst. Publ. 24, Springer, New York, 1992.

[17] W. R. Scott, Group Theory, Dover, New York, 1987.

[18] K. Szlachanyi, On field algebra construction, arXiv preprint 0806.0041.

Inst. of Math. "Simion Stoilow" of the Romanian Academy P.O. Box 1-764, RO-014700, Bucharest, Romania

University of Bucharest, Faculty of Mathematics and Computer Science, 14 Academiei St., RO-010014, Bucharest 1, Romania

E-mail address: sebastian.burciu@imar.ro

Department of Mathematics, University of Pennsylvania, David Rittenhouse Lab, 209 S. 33Rd St. Philadelphia, PA 19104

E-mail address: 1kadison@math.upenn.edu 